\title{
Assessment of postoperative quality of life following pylorus- preserving gastrectomy and Billroth-I distal gastrectomy in gastric cancer patients: results of the nationwide postgastrectomy syndrome assessment study
}

\author{
Junya Fujita · Masazumi Takahashi · Takashi Urushihara · Kazuaki Tanabe • \\ Yasuhiro Kodera · Takeyoshi Yumiba • Hideo Matsumoto • Akinori Takagane • \\ Chikara Kunisaki · Koji Nakada
}

Received: 26 August 2014/Accepted: 30 December 2014/Published online: 31 January 2015

(C) The International Gastric Cancer Association and The Japanese Gastric Cancer Association 2015

\begin{abstract}
Background Pylorus-preserving gastrectomy (PPG) is increasingly being used to treat early gastric cancer in the middle third of the stomach, with the hope of ameliorating postoperative dysfunction and improving quality of life (QOL). We evaluated symptoms of postgastrectomy syndrome (PGS) and QOL by means of a newly developed integrated questionnaire, the Postgastrectomy Syndrome Assessment Scale-45 (PGSAS-45), and compared PPG with Billroth-I distal gastrectomy (DGBI).

Methods The PGSAS-45 consists of 45 items, including items from the SF-8 and GSRS instruments, as well as 22 newly selected items. It was designed to assess the severity of PGS and the living status and QOL of gastrectomized patients. The nationwide PGSAS surveillance study enrolled 2,368 gastric cancer patients who underwent
\end{abstract}

\section{J. Fujita ( $\square)$}

Department of Surgery, NTT West Osaka Hospital, 2-6-40

Karasugatsuji Tennoji-ku, Osaka 543-8922, Japan

e-mail: j.fujita@mhc.west.ntt.co.jp

\section{Takahashi}

Division of Gastroenterological Surgery, Yokohama Municipal

Citizen's Hospital, Yokohama, Japan

\section{T. Urushihara}

Department of Gastroenterological, Breast and Transplant

Surgery, Hiroshima Prefectural Hospital, Hiroshima, Japan

\section{K. Tanabe}

Department of Gastroenterological and Transplant Surgery, Institute of Biomedical and Health Sciences, Hiroshima

University, Hiroshima, Japan

\section{Y. Kodera}

Department of Gastroenterological Surgery, Nagoya University

Graduate School of Medicine, Nagoya, Japan various types of gastrectomy. In this study we analyzed 313 PPG patients and 909 DGBI patients.

Results Body weight loss was $-6.9 \%$ in the PPG group and $-7.9 \%$ in the DGBI group $(P=0.052)$. The PPG group scored better on the diarrhea subscale (PPG; 1.8 vs. DGBI; $2.1, P<0.0001)$, dumping subscale (1.8 vs. 2.0 , $P=0.003)$, and frequency of additional meals (1.8 vs. 1.9 , $P=0.034)$. Multiple regression analysis revealed that age and the preservation of the celiac branch of the vagus nerve were independent factors predicting diarrhea and dumping. Conclusions It has been suggested that PPG is superior to DGBI for ameliorating PGS. Preservation of the celiac branch of the vagus nerve is recommended to reduce postoperative disorders regardless of the reconstruction method used.

Keywords PPG $\cdot$ DGBI $\cdot$ QOL $\cdot$ PGSAS-45

\section{T. Yumiba}

Department of Surgery, Kenporen Osaka Central Hospital,

Osaka, Japan

H. Matsumoto

Department of Digestive Surgery, Kawasaki Medical School, Kurashiki, Japan

\section{A. Takagane}

Department of Surgery, Hakodate Goryoukaku Hospital,

Hakodate, Japan

C. Kunisaki

Department of Surgery, Gastroenterological Center, Yokohama

City University Medical Center, Yokohama, Japan

K. Nakada

Department of Surgery, The Jikei University School of

Medicine, Tokyo, Japan 


\section{Introduction}

The radical surgical treatment of gastric cancer requires resection of a large portion of the stomach as well as regional lymphadenectomy. It is well known that gastric resection and reconstruction of the gastrointestinal tract result in a variety of functional and physiological disorders. These unpleasant alimentary and/or systemic symptoms are collectively referred to as postgastrectomy syndrome (PGS) [1, 2]. A recent report from the Japanese nationwide gastric cancer registry showed that patients with early gastric cancer (EGC) accounted for approximately $60 \%$ of all gastric cancer patients who underwent gastrectomy, and the 5-year survival rate of EGC patients exceeded $90 \%$ [3]. It is important to determine which gastrectomy operations can most minimize PGS, as EGC patients can survive for a long period of time following surgery.

The Billroth-I (BI) method is one of the most commonly performed reconstruction procedures following distal gastrectomy (DG) in gastric cancer patients. In Japan, the number of surgeons who prefer Roux-en-Y (RY) anastomosis has been increasing recently; however, the majority of medical institutions still select BI for reconstruction after DG (DGBI). The questionnaire survey regarding the reconstruction methods after gastrectomy conducted in Japan showed that BI was selected as the first choice for DG in $77 \%$ of Japanese institutions, RY in $21 \%$, and BII in $0.7 \%$ [4]. The operative simplicity and physiological passage of the diet through the duodenum are regarded as merits of DGBI, although postoperative reflux symptoms, esophagitis, and remnant gastritis were found to be significantly more frequent in BI patients compared with those receiving $\mathrm{RY}$, indicating that the bile reflux, which is attributed to the direct anastomosis between the gastric stump and duodenum, is one of the disadvantages of DGBI [5, 6].

Pylorus-preserving gastrectomy (PPG) was initially devised for resection of gastric ulcers by Maki and colleagues in 1967 [7], but it has recently been applied to EGC patients with the intention of attenuating PGS by maintaining pyloric function [8, 9]. With accumulation of data demonstrating acceptable long-term survival following PPG in EGC patients [10-12], the Japanese gastric cancer treatment guidelines (version 3) recognized PPG as an alternative to conventional DGBI for cT1N0 tumors located in the middle third of the stomach [13]. It is important to determine whether PPG is superior to DGBI in terms of comprehensively ameliorating PGS and improving QOL after gastrectomy.

The postgastrectomy syndrome assessment scale-45 (PGSAS-45) was newly developed by the Japanese Postgastrectomy Syndrome Working Party (JPGSWP) as an integrated questionnaire designed to assess postgastrectomy-specific clinical symptoms and QOL [14]. The
JPGSWP also conducted multiinstitutional nationwide surveillance to investigate long-term symptoms, living status and QOL following various types of gastrectomy. In the present study we used the PGSAS-45 to compare PPG and DGBI and evaluate the advantages of PPG as a function-preserving gastrectomy technique.

\section{Patients and methods}

Patients

Patient eligibility criteria were: (1) diagnosis of pathologically confirmed stage IA or IB gastric cancer; (2) first-time gastrectomy status; (3) age $\geq 20$ and $\leq 75$ years; (4) no history of chemotherapy; (5) no recurrence or distant metastasis; (6) gastrectomy conducted 1 or more years prior to enrollment date; (7) performance status (PS) $\leq 1$ on the Eastern Cooperative Oncology Group (ECOG) scale; (8) full capacity to understand and respond to the questionnaire; (9) no history of other diseases or surgeries that might influence responses to the questionnaire; (10) no presence of organ failure or mental illness; (11) written informed consent. Patients with dual malignancies or with concomitant resection of other organs (with co-resection equivalent to cholecystectomy being the exception) were excluded.

\section{QOL assessment}

The PGSAS-45 is an integrated questionnaire to assess the living status and the QOL in postgastrectomy patients, as described previously [14]. It consists of eight items from the Short-Form Health Survey (SF-8), 15 items from the Gastrointestinal Symptom Rating Scale (GSRS), and 22 items originally proposed by the JPGSWP members (Table 1). The items of the PGSAS-45 were classified into three domains, i.e., the symptom, living status, and QOL domains. The QOL domain consists of SF-8 and three of the dissatisfaction items; the living status domain consists of meal-associated items and ability to work. The symptom domain is composed of 23 symptom-related items clustered into seven symptom subscales (SS), i.e., the esophageal reflux $\mathrm{SS}$, abdominal pain $\mathrm{SS}$, meal-related distress $\mathrm{SS}$, indigestion SS, diarrhea SS, constipation SS, and dumping SS. In items 1-8, 34, 35, and 38-40, higher scores indicate better outcomes; in items 9-28, 30, 31, 33, and 41-45, higher scores indicate worse outcomes.

Study methods

This is a retrospective cross-sectional study conducted by continuous sampling from a central registration system for 
Table 1 Structure of postgastrectomy syndrome assessment scale-45 (PGSAS-45) (domains/subdomains/items/subscales)

\begin{tabular}{|c|c|c|c|}
\hline Domains & Subdomains & Items & Subscales \\
\hline QOL & SF-8 (QOL) & $\begin{array}{l}1 \text { Physical functioning* } \\
2 \text { Role physical* } \\
3 \text { Bodily pain* } \\
4 \text { General health* } \\
5 \text { Vitality* } \\
6 \text { Social functioning* } \\
7 \text { Role emotional* } \\
8 \text { Mental health* }\end{array}$ & $\begin{array}{l}\text { Physical component summary* (items 1-8) } \\
\text { Mental component summary* (items 1-8) }\end{array}$ \\
\hline Symptoms & GSRS (symptoms) & $\begin{array}{l}9 \text { Abdominal pains } \\
10 \text { Heartburn } \\
11 \text { Acid regurgitation } \\
12 \text { Sucking sensations in the epigastrium } \\
13 \text { Nausea and vomiting } \\
14 \text { Borborygmus } \\
15 \text { Abdominal distension } \\
16 \text { Eructation } \\
17 \text { Increased flatus } \\
18 \text { Decreased passage of stool } \\
19 \text { Increased passage of stool } \\
20 \text { Loose stool } \\
21 \text { Hard stool } \\
22 \text { Urgent need for defecation } \\
23 \text { Feeling of incomplete evacuation }\end{array}$ & $\begin{array}{l}\text { Esophageal reflux subscale (items } 10,11,13,24 \text { ) } \\
\text { Abdominal pain subscale (items } 9,12,28 \text { ) } \\
\text { Meal-related distress subscale (items 25-27) } \\
\text { Indigestion subscale (items } 14-17 \text { ) } \\
\text { Diarrhea subscale (items } 19,20,22 \text { ) } \\
\text { Constipation subscale (items } 18,21,23 \text { ) } \\
\text { Dumping subscale (items } 30,31,33 \text { ) } \\
\text { Total symptom score (above seven subscales) }\end{array}$ \\
\hline & Symptoms & $\begin{array}{l}24 \text { Bile regurgitation } \\
25 \text { Sense of food sticking } \\
26 \text { Postprandial fullness } \\
27 \text { Early satiation } \\
28 \text { Lower abdominal pain } \\
29 \text { Number and type of early dumping symptoms } \\
30 \text { Early dumping general symptoms } \\
31 \text { Early dumping abdominal symptoms } \\
32 \text { Number and type of late dumping symptoms } \\
33 \text { Late dumping symptoms }\end{array}$ & \\
\hline Living status & Meals (amount) 1 & $\begin{array}{l}34 \text { Ingested amount of food per meal* } \\
35 \text { Ingested amount of food per day* } \\
36 \text { Frequency of main meals } \\
37 \text { Frequency of additional meals }\end{array}$ & \\
\hline & Meals (quality) & $\begin{array}{l}38 \text { Appetite* } \\
39 \text { Hunger feeling* } \\
40 \text { Satiety feeling* }\end{array}$ & Quality of ingestion subscale* (items 38-40) \\
\hline & $\begin{array}{l}\text { Meals (amount) } 2 \\
\text { Social activity }\end{array}$ & $\begin{array}{l}41 \text { Necessity for additional meals } \\
42 \text { Ability to work }\end{array}$ & \\
\hline QOL & Dissatisfaction (QOL) & $\begin{array}{l}43 \text { Dissatisfaction with symptoms } \\
44 \text { Dissatisfaction at the meals } \\
45 \text { Dissatisfaction at working }\end{array}$ & Dissatisfaction for daily life subscale (items $43-45$ ) \\
\hline
\end{tabular}

PGSAS-45 postgastrectomy syndrome assessment scale-45, SF-8 short form-8, QOL quality of life, GSRS gastrointestinal symptom rating scale. In items or subscales with *, higher scores indicate better conditions. In items or subscales without*, higher scores indicate worse conditions. Each subscale is calculated as the mean of its composite items or subscales, except the physical and mental component summaries of SF-8. Items 29 and 32 do not have scores; these items were analyzed separately 
participant enrollment. The questionnaire was distributed to all eligible patients as they presented to the participating clinics, and patients were instructed to send the forms directly by mail to the data center. All QOL data from the questionnaires were matched with individual patient data collected via case report forms. The postoperative body weight was measured at the time of enrollment in the study.

Between July 2009 and December 2010, a total of 2,922 patients from 52 institutions were enrolled in the PGSAS surveillance study (Fig. 1). A total of 2,520 (86 \%) answers were retrieved, but of these 152 cases were excluded because of: age $\geq 76$ years $(n=90)$, postoperative period $<1$ year $(n=29)$, concomitant resection of other organs $(n=8)$, and other factors $(n=25)$. As a result, 2,368 $(81 \%)$ patients were determined to be eligible for the inclusion criteria. Among the eligible 2,368 responders, 393 patients underwent total gastrectomy, 909 DGBI, 475 DGRY, 313 PPG, 193 proximal gastrectomy, and 85 local gastric resection. In the present study, 909 patients who underwent DGBI and 313 patients who underwent PPG were subjected to the analysis.

This study was registered with the University Hospital Medical Information Network's Clinical Trials Registry (UMIN-CTR; registration no. 000002116). The study protocol was approved by the institutional review board at each of the participating institutions. Written informed consent was obtained from all enrolled patients.

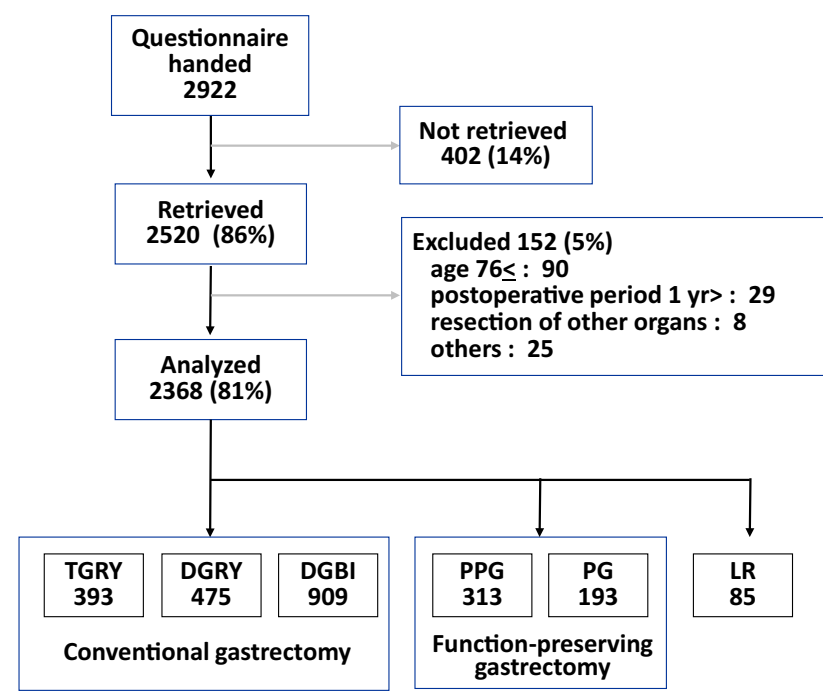

Fig. 1 Outline of the study. $T G$ Total gastrectomy with Roux-en-Y reconstruction, $D G R Y$ distal gastrectomy with Roux-en-Y reconstruction, $D G B I$ distal gastrectomy with Billroth I reconstruction, $P P G$ pylorus-preserving gastrectomy, $P G$ proximal gastrectomy, $L R$ local resection
Statistics

Statistical analyses were performed by the biostatisticians mainly using StatView for Windows, version 5.0 (SAS Institute, Inc.). Statistical methods included the $t$ test and chi-squared test. In the case of $P<0.1$ by univariate analysis, Cohen's $d$ was calculated. Main outcome measures that exhibited significant variations in univariate analysis were further analyzed using multiple regression analysis; $P<0.05$ was considered statistically significant. In the case of $P<0.1$ in multiple regression analysis, a standardization coefficient of regression $(\beta)$, decision coefficient $\left(R^{2}\right)$, and $P$ value are shown in Table 4 . Cohen's $d, \beta$, and $R^{2}$ measure effect sizes. Interpretation of effect sizes were $\geq 0.2$ small, $\geq 0.5$ medium, and $\geq 0.8$ large in Cohen's $d ; \geq 0.1$ small, $\geq 0.3$ medium, and $\geq 0.5$ large in $\beta ; \geq 0.02$ small, $\geq 0.13$ medium, and $\geq 0.26$ large in $R^{2}$.

\section{Results}

Patients characteristics

The patient characteristics are shown in Table 2. In the patient backgrounds there were no significant differences between the two groups except for gender where the proportion of males was higher in the DGBI group. In terms of the approach of surgery, the ratio of laparoscopic operations was similar in the two groups. The more extensive lymphadenectomy was conducted in DGBI patients compared with PPG patients, namely, almost all of the patients in the PPG group underwent $\leq \mathrm{D} 1$ dissection, while $36 \%$ of the patients in the DGBI group underwent D2 dissection $(P<0.0001)$. The celiac branch of the vagus nerve was preserved in $14.6 \%$ in DGBI and $68.0 \%$ in PPG $(P<0.0001)$. The size of the remnant stomach was significantly larger in the PPG than in the DGBI patients. The mean of the postoperative period at the time of enrollment in this study was 40.7 months and 38.4 months for DGBI and PPG, respectively.

\section{Main outcome measures}

Seven symptom SSs, the total symptom score, items representing the amount of food, quality of ingestion SS, items and SS representing dissatisfaction with life, the physical component summary (PCS) and mental component summary (MCS) from the PGSAS-45, and change in body weight at the enrollment were selected as the main outcome measures in this study based on the results of the validation analysis of PGSAS-45 as reported previously (Table 3) [14]. The results of the univariate analysis of the 
Table 2 Patient characteristics

\begin{tabular}{|c|c|c|c|}
\hline & DGBI & PPG & $P$ value \\
\hline Number of patients & 909 & 313 & \\
\hline $\begin{array}{l}\text { Postoperative period } \\
\text { (months) }\end{array}$ & $40.7+30.7^{\mathrm{a}}$ & $38.4+27.7$ & $\geq 0.1$ \\
\hline Age & $61.6+9.1^{\mathrm{a}}$ & $61.5+8.7^{\mathrm{a}}$ & $\geq 0.1$ \\
\hline \multicolumn{4}{|l|}{ Gender } \\
\hline Male & 594 & 183 & 0.0426 \\
\hline Female & 311 & 126 & \\
\hline Preoperative $\mathrm{BMI}^{\mathrm{b}}$ & $22.7+3.0^{\mathrm{a}}$ & $22.7+3.0^{\mathrm{a}}$ & $\geq 0.1$ \\
\hline Postoperative $\mathrm{BMI}^{\mathrm{b}}$ & $20.9+2.8^{\mathrm{a}}$ & $21.1+2.6^{\mathrm{a}}$ & $\geq 0.1$ \\
\hline \multicolumn{4}{|l|}{ Approach } \\
\hline Open & 489 & 173 & $\geq 0.1$ \\
\hline Laparoscopic & 415 & 136 & \\
\hline \multicolumn{4}{|c|}{ Extent of lymph node dissection ${ }^{c}$} \\
\hline D1> & 4 & 39 & $<0.0001$ \\
\hline D1 & 8 & 6 & \\
\hline D1a & 119 & 51 & \\
\hline D1b & 444 & 201 & \\
\hline D2 & 319 & 8 & \\
\hline \multicolumn{4}{|c|}{ Celiac branch of the vagal nerve } \\
\hline Preserved & 133 & 213 & $<0.0001$ \\
\hline Divided & 754 & 87 & \\
\hline \multicolumn{4}{|l|}{ Combined resection } \\
\hline None & 743 & \multicolumn{2}{|c|}{0.0057} \\
\hline Gallbladder & 80 & 12 & \\
\hline Spleen & 0 & 0 & \\
\hline Miscellaneous & 4 & 0 & \\
\hline \multicolumn{4}{|l|}{ Size of remnant stomach } \\
\hline Over half & 29 & 73 & \\
\hline Around one-third & 799 & 222 & \\
\hline Less than one-quarter & 61 & 12 & \\
\hline
\end{tabular}

${ }^{a}$ Mean \pm SD

b Body mass index

c According to the Japanese gastric cancer treatment guidelines

main outcome measures comparing DGBI with PPG are shown in Table 3. Regarding the symptom domain, PPG patients had significantly lower scores (indicating better conditions) in the diarrhea and dumping SSs, although there were no differences in the other symptom SSs or in the total symptom score between the two groups. The loss of body weight was marginally greater for DGBI than PPG, $-7.9 \%$ and $-6.9 \%$, respectively $(P=0.052)$. Although the ingested amount of food per meal was not different in the two groups, the necessity for an additional meal was statistically higher in the DGBI group compared with the PPG group $(P=0.034)$. There were no differences in the quality of ingestion, dissatisfactions categorized in the QOL domain, or the PCS and MCS of SF-8 between the two groups.

Next we conducted a multiple regression analysis for the diarrhea SS, dumping SS, and necessity for an additional meal by adding the type of gastrectomy, postoperative period, age, gender, surgical approach, and celiac branch preservation as explanatory variables (Table 4). Younger age and division of the celiac branch were detected as common and significant independent factors predicting diarrhea and dumping, but the type of gastrectomy was not. Female gender and younger age were detected as a significant independent factor for deteriorating dumping. As for living status, male gender, a longer postoperative period, a laparoscopic approach, PPG and younger age acted independently to reduce the necessity for an additional meal.

Outcome measures of singular symptom items

The results of each singular score among GSRS and PGSAS-45 specific symptom items are shown in Table 5. All of the component items of the diarrhea SS, namely increased passage of stools, loose stools, urgent need for defecation, and all of the component items of the dumping SS, namely early dumping general symptoms, early dumping abdominal symptoms, and late dumping symptoms, were significantly better in the PPG group compared with the DGBI group. The only worse score in PPG than DGBI was eructation $(P=0.0004)$.

\section{Discussion}

In recent years, PPG has been widely used in EGC patients as a function-preserving surgery that aims to reduce PGS and improve postoperative QOL. It is anticipated that preservation of the pyloric sphincter may attenuate rapid gastric emptying and suppress dumping symptomatology, which is one of the most unpleasant sequelae after gastrectomy and substantially affects QOL $[15,16]$. Thus far, several studies have used various methods to assess the superiority of PPG over DGBI for reducing dumping [1723]. We developed the PGSAS-45 as a comprehensive evaluation tool specific to PGS. It contains three items that evaluate the intensity of dumping symptoms: early dumping - general, early dumping - abdominal, and late dumping. In the current study we analyzed these outcomes in detail with a large number of patients and found that the scores for each of these three items were significantly lower in the PPG than in the DGBI group. Our results confirmed those of other investigators showing the superiority of PPG over DGBI in terms of dumping symptoms. In addition, we found that the PPG group scored 
Table 3 Univariate analysis of main outcome measures following distal gastrectomy (DGBI) and pylorus-preserving gastrecrtomy (PPG) procedures

\begin{tabular}{|c|c|c|c|c|c|c|c|c|}
\hline \multirow[t]{2}{*}{ Domains } & \multirow[t]{2}{*}{ Subdomains } & \multirow[t]{2}{*}{ Main outcome measures } & \multicolumn{2}{|l|}{ DGBI } & \multicolumn{2}{|l|}{ PPG } & \multicolumn{2}{|c|}{ Univariate analysis } \\
\hline & & & Mean & SD & Mean & SD & $P$-value & Cohen's $d$ \\
\hline \multirow[t]{7}{*}{ Symptoms } & PGSAS subscales & $\frac{\text { Esophageal reflux subscale }}{13,24)}$ (items 10,11 , & 1.7 & 0.8 & 1.7 & 0.8 & $\geq 0.1$ & \\
\hline & \multirow[t]{6}{*}{$\begin{array}{l}\text { (GSRS and } \\
\text { PGSAS items) }\end{array}$} & 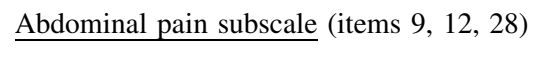 & 1.7 & 0.7 & 1.6 & 0.7 & $\geq 0.1$ & \\
\hline & & $\underline{\text { Meal-related distress subscale (items 25-27) }}$ & 2.1 & 0.9 & 2.1 & 0.9 & $\geq 0.1$ & \\
\hline & & Indigestion subscale (items 14-17) & 2.0 & 0.8 & 2.0 & 0.9 & $\geq 0.1$ & \\
\hline & & Diarrhea subscale (items 19, 20, 22) & 2.1 & 1.1 & 1.8 & 1.0 & $<.0001$ & 0.27 \\
\hline & & 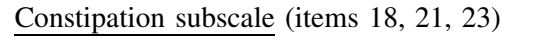 & 2.2 & 1.0 & 2.2 & 1.1 & $\geq 0.1$ & \\
\hline & & $\underline{\text { Dumping subscale }}$ (items $30,31,33$ ) & 2.0 & 1.0 & 1.8 & 0.9 & 0.003 & 0.21 \\
\hline Total & & $\underline{\text { Total symptom score }}$ & $\underline{2.0}$ & $\underline{0.7}$ & $\underline{1.9}$ & $\underline{0.7}$ & $\geq 0.1$ & \\
\hline Living & Body weight & Change in body weight & $-7.9 \%$ & $8.1 \%$ & $-6.9 \%$ & $7.0 \%$ & 0.052 & $(0.13)$ \\
\hline \multirow[t]{4}{*}{ Status } & \multirow[t]{2}{*}{ Meals(amount) } & Ingested amount of food per meal & 7.1 & 2.0 & 7.0 & 1.9 & $\geq 0.1$ & \\
\hline & & Necessity for additional meals & 1.9 & 0.8 & 1.8 & 0.8 & 0.034 & $(0.14)$ \\
\hline & Meals(quality) & $\frac{\text { Quality of ingestion subscale* }}{\text { (items } 38-40 \text { ) }}$ & 3.8 & 0.9 & 3.8 & 0.9 & $\geq 0.1$ & \\
\hline & $\underline{\text { Social activity }}$ & Ability to work & $\underline{1.8}$ & $\underline{0.9}$ & $\underline{1.8}$ & $\underline{0.9}$ & $\geqq 0.1$ & \\
\hline \multirow[t]{6}{*}{ QOL } & \multirow[t]{4}{*}{ Dissatisfaction } & Dissatisfaction with symptoms & 1.8 & 0.9 & 1.8 & 0.9 & $\geq 0.1$ & \\
\hline & & Dissatisfaction at the meal & 2.2 & 1.1 & 2.2 & 1.1 & $\geq 0.1$ & \\
\hline & & Dissatisfaction at working & 1.7 & 0.9 & 1.7 & 0.9 & $\geq 0.1$ & \\
\hline & & $\frac{\text { Dissatisfaction for daily life subscale }}{\text { (items } 43-45 \text { ) }}$ & 1.9 & 0.8 & 1.9 & 0.8 & $\geq 0.1$ & \\
\hline & \multirow[t]{2}{*}{ SF-8 } & $\frac{\text { Physical component summary (PCS)* }}{\text { (items } 1-8)}$ & 50.5 & 5.5 & 51.1 & 5.3 & $\geq 0.1$ & \\
\hline & & $\frac{\text { Mental component summary (MCS)* }}{\text { (items } 1-8)}$ & $\underline{49.9}$ & $\underline{5.7}$ & $\underline{50.0}$ & $\underline{6.1}$ & $\geq 0.1$ & \\
\hline \multicolumn{3}{|c|}{ The interpretation of effect size } & & & & & & $\underline{\text { Cohen's }} \underline{d}$ \\
\hline \multicolumn{3}{|c|}{ (None-very small) } & & & & & & $(<0.20)$ \\
\hline \multicolumn{2}{|l|}{ Small } & & & & & & & $\geq 0.20$ \\
\hline \multicolumn{2}{|l|}{ Medium } & & & & & & & $\geq 0.50$ \\
\hline \multicolumn{2}{|l|}{ Large } & & & & & & & $\geq 0.80$ \\
\hline
\end{tabular}

Integrated subscales are underlined in the table. Outcome measures with *, higher score indicating better condition. Outcome measures without *, higher score indicating worse condition. Each subscale is calculated as the mean of composed items or subscales, except PCS or MCS of SF-8

significantly lower on the diarrhea subscale of the PGSAS45 than the DGBI group. These results indicated that by preventing the rapid evacuation of the contents of the remnant stomach into the small intestine, PPG might reduce the incidence of increased passage of stool, loose stool, and urgent need for defecation.

A secondary advantage of PPG with regard to postoperative function is that it is thought to suppress duodenogastric reflux. It has been widely reported that the incidence of bile regurgitation and remnant gastritis is significantly lower in PPG than DGBI patients as assessed by endoscopic examination [15, 18, 24]. Nevertheless, in the present study there were no significant differences between the two groups in the scores of items associated with gastritis and bile reflux. Namely, the suppression of bile reflux by PPG did not decrease symptomatic complaints. Since the visceral sensation to chemical stimulants such as bile and acid is mediated primarily by vagal input, our findings may be explained at least in part by the loss of this input to the remnant stomach due to perigastric lymph node dissection. Even though there were no differences in symptoms between the PPG and DGBI groups, we still should pay attention to latent injuries to the remnant gastric mucosa. Long-term observations are needed to evaluate the histological influence of these procedures on the remnant stomach and any secondary carcinogenesis.

Another expected advantage of PPG is prandial and nutritional improvement. Reduced food intake and loss of 


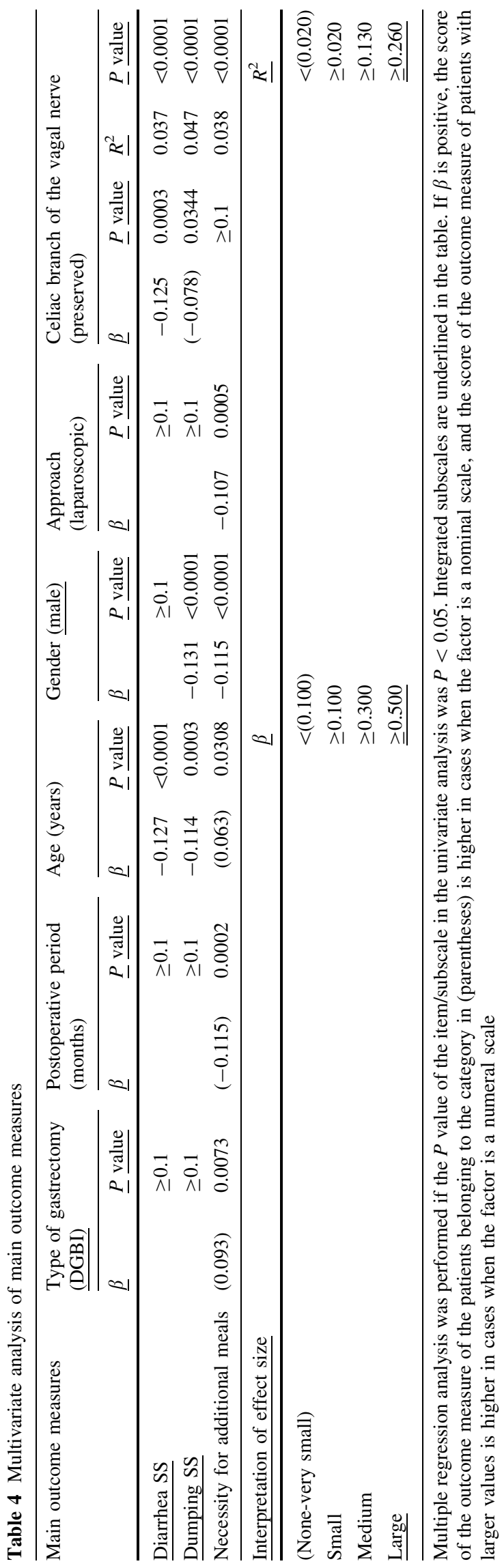

body weight greatly influence living status and QOL in patients who undergo gastrectomy. In general, PPG enables conservation of a relatively large gastric remnant compared to conventional DG. Because the present study was retrospective in design and we did not control the extent of gastric excision, the sizes of the remnant stomach differed significantly between the two groups. In the PPG group, at least half of the original stomach was preserved in $24 \%$ of patients, in contrast to only $3 \%$ of patients in the DGBI group. This may be the primary reason why PPG patients required fewer additional meals and why body weight loss was smaller in the PPG than the DGBI group ($6.9 \pm 7.0 \%$ vs. $-7.9 \pm 8.1 \%, P=0.052$ ). Namikawa et al. [25] used the PGSAS-45 to conduct a more detailed analysis of PPG patients and reported that larger proximal gastric remnants resulted in less body weight loss and dissatisfaction at meals and in daily life.

Despite these positive findings, a typical adverse outcome after PPG is delayed gastric emptying. This occurs significantly more frequently in the early postoperative period in PPG patients than in those undergoing DGBI and is generally recognized as a PPG-specific complication [26]. Food residue was frequently observed in the remnant stomach in PPG patients even several years after surgery [24], although this disorder gradually improves over time. We evaluated symptoms of delayed gastric emptying using the "postprandial fullness" and "early satiation" items of the PGSAS-45 and found no significant differences between the PPG and DGBI group for either item. As the average postoperative period of the PPG patients was 38.4 months, we consider that delayed gastric emptying caused by PPG resolved over this period of time.

Multiple regression analysis revealed that younger age and female gender were independent factors influencing PGSAS-45 symptom scores of diarrhea and dumping, and longer postoperative periods attenuated the need for additional meals. Taken together, these findings suggest that relatively young female EGC patients might be good candidates for function-preserving surgery that can minimize these postoperative disorders.

Recently, gastrectomy with preservation of the celiac branch of the vagus nerve has become widely used in EGC patients. The celiac branch innervates the upper gastrointestinal organs, such as the stomach, duodenum, jejunum, and pancreas, and some of these organs' physiological functions are regulated by the afferent-efferent network of the vagus nerve connecting the hypothalamus and visceral organs. In an experimental study using a canine model, Ando et al. [27] demonstrated that section of the celiac branch decreased the postprandial motility of the duodenum and jejunum. Other investigators also showed that denervation of the celiac branch influenced the regulation of hormonal secretion. Kinami et al. [28] reported that 
Table 5 Univariate analysis of other outcome measures (symptoms)

\begin{tabular}{|c|c|c|c|c|c|c|c|}
\hline \multirow[t]{3}{*}{ Subdomains } & \multirow[t]{3}{*}{ Items } & \multicolumn{6}{|c|}{ Univariate analysis } \\
\hline & & \multicolumn{3}{|l|}{ DGBI } & \multicolumn{3}{|l|}{ PPG } \\
\hline & & Mean & SD & Mean & SD & $P$ value & Cohen's $d$ \\
\hline \multirow[t]{15}{*}{ GSRS items } & Abdominal pain & 1.7 & 0.9 & 1.8 & 1.0 & $\geq 0.1$ & \\
\hline & Heartburn & 1.8 & 1.0 & 1.8 & 1.0 & $\geq 0.1$ & \\
\hline & Acid regurgitation & 1.8 & 1.1 & 1.8 & 1.1 & $\geq 0.1$ & \\
\hline & Sucking sensations in the epigastrium & 1.5 & 0.8 & 1.5 & 0.8 & $\geq 0.1$ & \\
\hline & Nausea and vomiting & 1.5 & 0.9 & 1.5 & 0.9 & $\geq 0.1$ & \\
\hline & Borborygmus & 1.8 & 1.0 & 1.7 & 1.0 & $\geq 0.1$ & \\
\hline & Abdominal distension & 2.0 & 1.1 & 2.0 & 1.1 & $\geq 0.1$ & \\
\hline & Eructation & 1.7 & 1.0 & 1.9 & 1.1 & 0.0004 & 0.22 \\
\hline & Increased flatus & 2.5 & 1.3 & 2.4 & 1.3 & $\geq 0.1$ & \\
\hline & Decreased passage of stools & 2.2 & 1.3 & 2.3 & 1.4 & $\geq 0.1$ & \\
\hline & Increased passage of stools & 2.1 & 1.3 & 1.9 & 1.2 & 0.0010 & 0.22 \\
\hline & Loose stools & 2.1 & 1.1 & 1.9 & 1.1 & 0.0098 & 0.17 \\
\hline & Hard stools & 2.0 & 1.1 & 2.1 & 1.2 & $\geq 0.1$ & \\
\hline & Urgent need for defecation & 2.2 & 1.3 & 1.8 & 1.1 & $<0.0001$ & 0.32 \\
\hline & Feeling of incomplete evacuation & 2.4 & 1.1 & 2.3 & 1.1 & $\geq 0.1$ & \\
\hline \multirow[t]{8}{*}{ PGSAS specific items } & Bile regurgitation & 1.8 & 1.0 & 1.7 & 0.9 & $\geq 0.1$ & \\
\hline & Sense of food sticking & 1.6 & 0.9 & 1.6 & 0.9 & $\geq 0.1$ & \\
\hline & Postprandial fullness & 2.3 & 1.2 & 2.4 & 1.2 & $\geq 0.1$ & \\
\hline & Early satiation & 2.3 & 1.1 & 2.4 & 1.1 & $\geq 0.1$ & \\
\hline & Lower abdominal pains & 1.8 & 1.0 & 1.7 & 1.0 & 0.0160 & 0.16 \\
\hline & Early dumping general symptoms & 1.9 & 1.2 & 1.7 & 1.1 & 0.0089 & 0.18 \\
\hline & Early dumping abdominal symptoms & 2.3 & 1.3 & 2.1 & 1.3 & 0.0333 & 0.14 \\
\hline & Late dumping symptoms & 1.8 & 1.1 & 1.5 & 1.0 & 0.0081 & 0.19 \\
\hline
\end{tabular}

section of the celiac branch during gastrectomy impaired the secretion of insulin in response to glucagon administration, and Takiguchi et al. [29] recently demonstrated that denervation of the celiac branch disturbed postprandial changes in ghrelin secretion. As described above, preservation of the celiac branch might be beneficial for maintaining physiologic functions, but the practical efficacy of this surgical procedure in gastrectomy has not been fully elucidated $[30,31]$. In the present study, $71 \%$ of PPG patients and $15 \%$ of DGBI patients underwent preservation of the celiac branch. Multivariable regression analysis revealed that preservation of the celiac branch significantly affected the dumping SS and diarrhea SS scores, although the method of reconstruction (PPG vs. DGBI) was not an independent factor predicting these symptoms. These findings strongly suggest that the celiac branch of the vagus nerve should be preserved whenever possible regardless of which reconstruction method is preferred.

In contrast to the differences in symptom scores between PPG and DGBI, we did not detect any differences between the two approaches in terms of general QOL scores on the PGSAS-45. One reason may be that the effect size of the difference for each symptom item was relatively small, represented as Cohen's $d$ values of $0.17-0.32$, which might result in a lack of total improvement in the general QOL scores. Similar findings were observed in other series of the PGSAS study, i.e., DGBI vs. DG Roux-en-Y [5] and total gastrectomy vs. proximal gastrectomy [32].

Our work has several limitations that may be attributed to the retrospective design of the study. The PPG surgical procedures conducted in this study were considerably lacking in uniformity; specifically, the following factors varied widely among institutions and surgeons: the extent of lymphadenectomy, size of the remnant stomach, length of the pyloric cuff, surgical approach (open or laparoscopic), method of anastomosis (hand sewn or stapling), and preservation of the vagus nerve and infrapyloric vessels. All of these factors are known to substantially influence postoperative dysfunction following PPG [33]. Another weakness of this study was the sizable variation in the postoperative period of the enrolled patients. Determining the optimal time point to evaluate the postoperative change of QOL properly is a matter of concern. In addition, there might also be a slight placebo effect, as the PPG 
patients probably were told that this operation was used with the intention to reduce postoperative symptoms. Future prospective controlled studies are desired that provide the most suitable uniform surgery in each study group and have appropriate timing for the assessment.

In conclusion, we demonstrated that PPG patients in this study had superior results compared to DGBI patients in terms of suppressing postoperative dumping symptoms, diarrhea, and the need for additional food. Preservation of the celiac branch of the vagus nerve is recommended either in DGBI or PPG for EGC.

Acknowledgments This study was supported by a grant from Jikei University and the Japanese Society for Gastro-Surgical Pathophysiology. The authors thank all physicians who participated in this study and the patients whose cooperation made it possible.

Conflict of interest The authors declare no conflicts of interest with regard to this manuscript.

\section{References}

1. Bolton JS, Conway WC 2nd. Postgastrectomy syndromes. The Surgical Clinics of North America. 2011;91(5):1105-22.

2. Carvajal SH, Mulvihill SJ. Postgastrectomy syndromes: dumping and diarrhea. Gastroenterol Clin North Am. 1994;23(2):261-79.

3. Nashimoto A, Akazawa K, Isobe Y, Miyashiro I, Katai H, Kodera Y, et al. Gastric cancer treated in 2002 in Japan: 2009 annual report of the JGCA nationwide registry. Gastric Cancer. 2013;16(1):1-27.

4. Kumagai K, Shimizu K, Yokoyama N, Aida S, Arima S, Aikou T. Questionnaire survey regarding the current status and controversial issues concerning reconstruction after gastrectomy in Japan. Surg Today. 2012;42(5):411-8.

5. Terashima M, Tanabe K, Yoshida M, Kawahira H, Inada $T$, Okabe $\mathrm{H}$, et al. Postgastrectomy syndrome assessment scale (PGSAS)-45 and changes in body weight are useful tools for evaluation of reconstruction methods following distal Gastrectomy. Ann Surg Oncol. 2014;21(Suppl 3):370-8.

6. Hirao M, Takiguchi S, Imamura H, Yamamoto K, Kurokawa Y, Fujita J, et al. Comparison of Billroth I and Roux-en-Y reconstruction after distal gastrectomy for gastric cancer: one-year postoperative effects assessed by a multi-institutional RCT. Ann Surg Oncol. 2013;20(5):1591-7.

7. Maki T, Shiratori T, Hatafuku T, Sugawara K. Pylorus-preserving gastrectomy as an improved operation for gastric ulcer. Surgery. 1967;61(6):838-45.

8. Kodama M, Koyama K. Indications for pylorus preserving gastrectomy for early gastric cancer located in the middle third of the stomach. World J Surg. 1991;15(5):628-33 (Discussion 33-34).

9. Isozaki H, Okajima K, Momura E, Ichinona T, Fujii K, Izumi N, et al. Postoperative evaluation of pylorus-preserving gastrectomy for early gastric cancer. Br J Sur. 1996;83(2):266-9.

10. Morita S, Katai H, Saka M, Fukagawa T, Sano T, Sasako M. Outcome of pylorus-preserving gastrectomy for early gastric cancer. Br J Surg. 2008;95(9):1131-5.

11. Hiki N, Sano T, Fukunaga T, Ohyama S, Tokunaga M, Yamaguchi T. Survival benefit of pylorus-preserving gastrectomy in early gastric cancer. J Am Coll Surg. 2009;209(3):297-301.

12. Matsuki A, Nashimoto A, Yabusaki H, Nakagawa S. Long-term clinical outcome and survival after pylorus-preserving gastrectomy. Hepatogastroenterology. 2012;59(118):2012-5.
13. Japanese Gastric Cancer Association. Japanese gastric cancer treatment guidelines 2010 (ver. 3). Gastric Cancer Off J Int Gastric Cancer Assoc Japan Gastric Cancer Assoc. 2011;14(2): 113-23.

14. Nakada K, Ikeda M, Takahashi M, Kinami S, Yoshida M, Uenosono Y, et al. Characteristics and clinical relevance of postgastrectomy syndrome assessment scale (PGSAS)-45: newly developed integrated questionnaires for assessment of living status and quality of life in postgastrectomy patients. Gastric Cancer. 2014;. doi:10.1007/s10120-014-0344-4.

15. Imada T, Rino Y, Takahashi M, Suzuki M, Tanaka J, Shiozawa $\mathrm{M}$, et al. Postoperative functional evaluation of pylorus-preserving gastrectomy for early gastric cancer compared with conventional distal gastrectomy. Surgery. 1998;123(2):165-70.

16. Nishikawa K, Kawahara H, Yumiba T, Nishida T, Inoue Y, Ito T, et al. Functional characteristics of the pylorus in patients undergoing pylorus-preserving gastrectomy for early gastric cancer. Surgery. 2002;131(6):613-24.

17. Nakane $\mathrm{Y}$, Akehira K, Inoue K, Iiyama H, Sato M, Masuya Y, et al. Postoperative evaluation of pylorus-preserving gastrectomy for early gastric cancer. Hepatogastroenterology. 2000;47(32): $590-5$.

18. Yamaguchi $\mathrm{T}$, Ichikawa $\mathrm{D}$, Kurioka $\mathrm{H}$, Ikoma $\mathrm{H}$, Koike $\mathrm{H}$, Otsuji E, et al. Postoperative clinical evaluation following pylorus-preserving gastrectomy. Hepatogastroenterology. 2004;51(57): 883-6.

19. Shibata C, Shiiba KI, Funayama Y, Ishii S, Fukushima K, Mizoi $\mathrm{T}$, et al. Outcomes after pylorus-preserving gastrectomy for early gastric cancer: a prospective multicenter trial. World J Surg. 2004;28(9):857-61.

20. Nunobe S, Sasako M, Saka M, Fukagawa T, Katai H, Sano T. Symptom evaluation of long-term postoperative outcomes after pylorus-preserving gastrectomy for early gastric cancer. Gastric Cancer. 2007;10(3):167-72.

21. de Park J, Lee HJ, Jung HC, Kim WH, Lee KU, Yang HK. Clinical outcome of pylorus-preserving gastrectomy in gastric cancer in comparison with conventional distal gastrectomy with Billroth I anastomosis. World J Surg. 2008;32(6):1029-36.

22. Mine S, Sano T, Tsutsumi K, Murakami Y, Ehara K, Saka M, et al. Large-scale investigation into dumping syndrome after gastrectomy for gastric cancer. J Am Coll Surg. 2010;211(5): 628-36.

23. Urushihara T, Sumimoto K, Shimokado K, Kuroda Y. Gastric motility after laparoscopically assisted distal gastrectomy, with or without preservation of the pylorus, for early gastric cancer, as assessed by digital dynamic x-ray imaging. Surg Endosc. 2004;18(6):964-8.

24. Nagano H, Ohyama S, Sakamoto Y, Ohta K, Yamaguchi T, Muto $\mathrm{T}$, et al. The endoscopic evaluation of gastritis, gastric remnant residue, and the incidence of secondary cancer after pyloruspreserving and transverse gastrectomies. Gastric Cancer. 2004;7(1):54-9.

25. Namikawa T, Hiki N, Kinami S, Okabe H, Urushihara T, Kawahira $\mathrm{H}$, et al. Factors that minimize postgastrectomy symptoms following pylorus-preserving gastrectomy: assessment using a newly developed scale (PGSAS-45). Gastric Cancer. 2014;. doi:10.1007/s10120-014-0366-y.

26. Suh YS, Han DS, Kong SH, Kwon S, Shin CI, Kim WH, et al. Laparoscopy-assisted pylorus-preserving gastrectomy is better than laparoscopy-assisted distal gastrectomy for middle-third early gastric cancer. Ann Surg. 2014;259(3):485-93.

27. Ando H, Mochiki E, Ohno T, Kogure N, Tanaka N, Tabe Y, et al. Effect of distal subtotal gastrectomy with preservation of the celiac branch of the vagus nerve to gastrointestinal function: an experimental study in conscious dogs. Ann Surg. 2008;247(6): 976-86. 
28. Kinami S, Miwa K, Sato T, Miyazaki I. Section of the vagal celiac branch in man reduces glucagon-stimulated insulin release. J Auton Nerv Syst. 1997;64(1):44-8.

29. Takiguchi S, Hiura Y, Takahashi T, Kurokawa Y, Yamasaki M, Nakajima K, et al. Preservation of the celiac branch of the vagus nerve during laparoscopy-assisted distal gastrectomy: impact on postprandial changes in ghrelin secretion. World J Surg. 2013;37(9):2172-9.

30. Tokunaga M, Hiki N, Fukunaga T, Ohyama S, Nunobe $\mathrm{S}$, Yamada K, et al. Is preservation of the celiac branch of the vagal nerve effective in preventing stasis following pylorus-preserving gastrectomy? Hepatogastroenterology. 2011;58(107-108): 1046-50.
31. Yamada H, Kojima K, Inokuchi M, Kawano T, Sugihara K. Efficacy of celiac branch preservation in Roux-en-y reconstruction after laparoscopy-assisted distal gastrectomy. Surgery. 2011;149(1):22-8.

32. Takiguchi $\mathbf{N}$, Takahashi $\mathbf{M}$, Ikeda $\mathrm{M}$, Inagawa $\mathrm{S}$, Ueda $\mathrm{S}$, Nobuoka $\mathrm{T}$, et al. Long-term quality-of-life comparison of total gastrectomy and proximal gastrectomy by Postgastrectomy Syndrome Assessment Scale (PGSAS-45): a nationwide multiinstitutional study. Gastric Cancer. 2014;. doi:10.1007/s10120014-0377-8.

33. Hiki N, Nunobe S, Kubota T, Jiang X. Function-preserving gastrectomy for early gastric cancer. Ann Surg Oncol. 2013;20(8):2683-92. 